\title{
Limit Analysis of Slope Stability by Rigid Finite-Element Method and Linear Programming Considering Rotational Failure
}

\author{
Fengtao $\mathrm{Liu}^{1}$ and Jidong Zhao ${ }^{2}$
}

\begin{abstract}
The authors present a numerical limit analysis on the slope stability in this paper using the rigid finite-element method (RFEM). The novelty of this study is the consideration of the rotational component of the centroid velocity for each element, as well as a generalized overturning failure criterion governing the element rotation. By combining the generalized rotation failure criterion with the Mohr-Coulomb failure criterion, the RFEM-based upper and lower bound limit analysis is formulated as a typical primal and dual linear programming problem and is solved effectively by a primal-dual interior-point method. The proposed formulation and methodology are validated by three classical soil or rock slope stability problems. Numerical results confirm the necessity of considering the rotation mechanism in RFEM-based limit analysis for slope stability in order to achieve realistic predictions. DOI: 10.1061/(ASCE)GM.1943-5622.0000283. @ 2013 American Society of Civil Engineers.
\end{abstract}

CE Database subject headings: Slope stability; Limit analysis; Finite element method; Failures.

Author keywords: Slope stability; Limit analysis; Rigid finite-element method; Primal-dual interior-point method; Rotation failure criterion.

\section{Introduction}

Landslides are one of the major natural hazards that greatly threaten human life and property in many countries. Great effort has thus been paid around the world every year to monitoring and analyzing the stability of soil and rock slopes. The limit equilibrium method (LEM; Ahmed et al. 2012) and limit analysis based on the upper and lower bound theorems have been widely used for slope stability analysis and design. In both of these approaches to slope stability analysis, two classical types of failure mechanisms have frequently been assumed, namely, sliding and rotational failures. Although the planar or circular sliding failure mechanism has commonly been adopted in many studies, rotational failure can be the key mechanism controlling the failure of a slope in some cases. For example, in analyzing the failure of the north face of the Vajont slide, Müller (1968) suggested that block rotation or toppling may have been a contributing factor. Chen (1975) also remarked that, in the limit equilibrium analysis of a uniform soil slope, most critical slip surfaces have been based on the assumption of a rigid body rotation failure mechanism with such surface shape as logarithmic spiral. Indeed, toppling failure may be a more important failure mode in the case of a rock slope. Following the earliest mathematical solution of toppling proposed by Goodman and Bray (1976), Zanbak (1983)

\footnotetext{
${ }^{1}$ Lecturer, Dept. of Earth Science, Sun Yat-Sen Univ., Xingang Xilu No. 135, Guangzhou, China, 510275; formerly, Postdoctoral Fellow, Dept. of Civil and Environmental Engineering, Hong Kong Univ. of Science and Technology, Clear Water Bay, KLN, Hong Kong (corresponding author). E-mail: celiuft@gmail.com

${ }^{2}$ Assistant Professor, Dept. of Civil and Environmental Engineering, Hong Kong Univ. of Science and Technology, Clear Water Bay, KLN, Hong Kong. E-mail: jzhao@ust.hk

Note. This manuscript was submitted on May 27, 2012; approved on December 27, 2012; published online on December 29, 2012. Discussion period open until May 1, 2014; separate discussions must be submitted for individual papers. This paper is part of the International Journal of Geomechanics, Vol. 13, No. 6, December 1, 2013. @ASCE, ISSN 1532$3641 / 2013 / 6-827-839 / \$ 25.00$.
}

constructed a set of diagrams for the calculation of the support force against toppling with different ratios of slope height to block thickness. Cruden (1989) further extended this model to include the effect of the friction angle along the block interfaces and the inclination of the slope. Some more recent studies have been devoted to refining the analytical solutions for block toppling (Liu et al. 2008, 2009) and flexural toppling (Amini et al. 2009).

Limit analysis based on upper and lower bound theorems has proved to be more robust and efficient than the conventional LEM in many situations, especially when it is combined with the power of modern computers. Since the pioneering work by Drucker and Prager (1952) and Chen (1975), there has been much progress in applying numerical limit analysis to geotechnical engineering and slope stability analysis (Donald and Chen 1997; Chen et al. 2001a, b). In particular, FEM based numerical limit analysis has been the prevailing method used by many researchers. Yu et al. (1998) and Kim et al. $(1999,2002)$ have applied the lower and upper bound approach proposed by Sloan $(1988,1989)$ and Sloan and Kleeman (1995) to evaluate the stability of slopes. Yang et al. (2004a, b) and Yang and Zou (2006), based on the Hoek-Brown criterion, proposed a tangential strength method in an upper bound analysis to obtain the stability factor of rock slopes. Li et al. (2008, 2009, 2011) have produced stability charts for rock slopes using the FEM-based upper and lower techniques proposed by Lyamin and Sloan (2002a, b) and Krabbenhoft et al. (2005). An obvious drawback with the traditional FEM-based limit analysis approaches being applied to rock slopes is their inability to characterize the behavior of discontinuous rock masses. In this regard, a good alternative would be the rigid FEM (RFEM; Kawai 1978; Qian and Zhang 1995). With a lower degree of freedom for the elements considered in the RFEM than that in the traditional FEM, the computational efficiency may be much improved. Importantly, the physical discontinuities in discontinuous media can be reasonably treated as interfaces between the adjacent rigid elements in RFEM. Based on RFEM, lower bound (Zhang and Qian 1993; Zhang 1999) and upper bound (Chen et al. 2003, 2004, 2005; Yang and Yang 2010) limit analysis methods have been developed.

In existing studies by RFEM, the constraint of compatibility between rigid elements has been imposed in a simplified way, which 
leads to the ignorance of rotational components of the centroid velocity of each rigid element (Zhang 1999; Chen et al. 2003, 2004, 2005). This may essentially lead to the exclusion of the rotational or toppling failure mode in numerical limit analysis, which, in some cases, may cause erroneous predictions as mentioned before. The authors present a new method in this paper by both keeping the compatibility condition between rigid elements and considering the rotational velocity for each element. The overturning criterion proposed by Goodman and Bray (1976) is generalized to govern the rotational failure. The formulations are then integrated into the RFEM-based limit analysis. Specifically, the discontinuous kinematically admissible velocity between two adjacent rigid elements in a typical upper bound analysis can now develop into two failure modes, namely, the simple sliding failure, which is controlled by the Mohr-Coulomb criterion, and the rotational failure, which is controlled by the new overturning criterion. A mixed formulation of limit analysis is then developed in which the compatibility equation and the equilibrium equation are enforced to be satisfied simultaneously, and this is then solved as a linear programming problem by an efficient primal-dual interior-point method proposed by Andersen et al. (1996). Finally, the present method is validated by several examples of soil and rock slope stability problems.

\section{Numerical Discretization Based on Rigid Finite Elements}

The following assumptions are generally taken in a RFEM: (1) All elements are assumed to be rigid and are connected to one another by the element interface. (2) The deformation energy of a system is stored in the interfaces only, and a discontinuous velocity field is allowed at the interface. (3) The interfaces are assumed to be isotropic, and their deformation is perfectly plastic, obeying the MohrCoulomb yield condition and the associated flow rule. With these assumptions, the compatibility and equilibrium equations between rigid elements can be found as follows.

\section{Compatibility Equation of Discontinuous Kinematical Admissible Velocity Field}

A possible discontinuous kinematical admissible velocity field at the interface is shown in Fig. 1 where $P_{m}$ denotes the center of the $k$ th interface between two typical rigid elements $i$ and $j$. The discontinuous velocity can be conveniently measured by a strain rate vector as follows:

$$
\dot{\boldsymbol{\varepsilon}}_{k}=\left[\begin{array}{lll}
\dot{n}_{k} & \dot{s}_{k} & \dot{\theta}_{k}
\end{array}\right]^{T}
$$

where $\dot{n}_{k}, \dot{s}_{k}$, and $\dot{\theta}_{k}=$ relative tangential, normal, and angular displacement rates, at the center of the joint, respectively. The discontinuous strain rate vectors at all interfaces of a discretized RFEM domain can be assembled into the following vector:

$$
\dot{\boldsymbol{\varepsilon}}^{T}=\left[\begin{array}{llll}
\dot{\boldsymbol{\varepsilon}}_{1}^{T} & \dot{\boldsymbol{\varepsilon}}_{2}^{T} & \ldots & \dot{\boldsymbol{\varepsilon}}_{n_{d}}^{T}
\end{array}\right]
$$

where $n_{d}=$ number of all interfaces.

In local coordinates $s_{k}^{i}$-o- $n_{k}^{i}$, the velocity at the center $P_{m}^{i}$ of the $k$ th interface caused by the centroid velocity of $i$ th element can be written as

$$
\dot{\boldsymbol{\varepsilon}}_{i / k}=\mathbf{L}_{i / k} \mathbf{N}_{i / k} \dot{\mathbf{u}}_{i}
$$

where $\dot{\mathbf{u}}_{i}=\left[\begin{array}{ccc}\dot{u}_{g}^{i} & \dot{v}_{g}^{i} & \dot{\omega}_{g}^{i}\end{array}\right]^{T}=$ centroid velocity of the $i$ th element; $\dot{u}_{g}^{i}$ and $\dot{v}_{g}^{i}=$ translational velocities in the $x$-and $y$-directions of the global coordinates $x$-o- $y$; $\dot{\omega}_{g}^{i}=$ rotational velocity of the $i$ th element; $\mathbf{N}_{i / k}=$ RFEM shape function of the $i$ th element corresponding to the $k$ th interface; and $\mathbf{L}_{i / k}=$ matrix of direction cosines of the local $s_{k}^{i}-\mathrm{o}-n_{k}^{i}$ axes for the $k$ th interface with respect to the global coordinate system $x$-o-y. Specifically, $\mathbf{N}_{i / k}$ and $\mathbf{L}_{i / k}$ are formulated as follows:

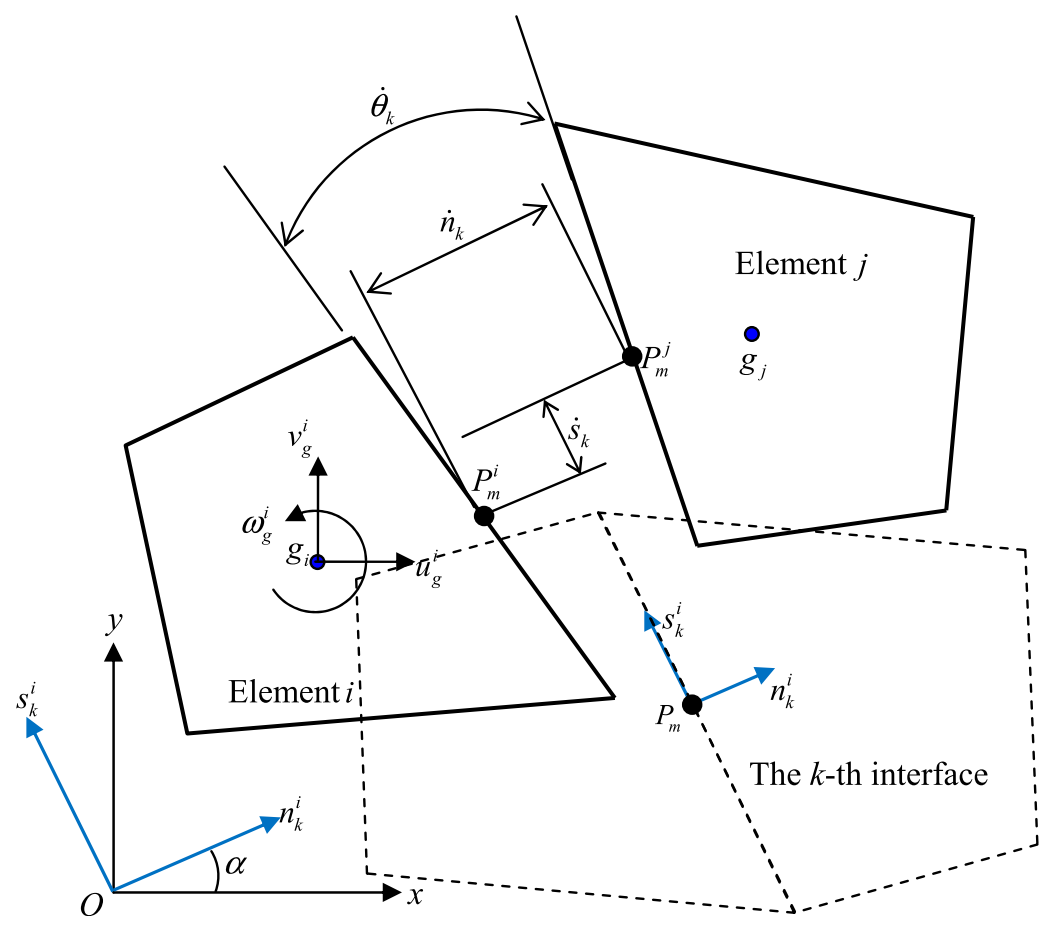

Fig. 1. Kinematically admissible discontinuous velocity field of interface between two rigid elements 


$$
\begin{gathered}
\mathbf{N}_{i / k}=\left[\begin{array}{ccc}
1 & 0 & y_{i / g}-y_{k / m} \\
0 & 1 & x_{k / m}-x_{i / g} \\
0 & 0 & 1
\end{array}\right] \\
\mathbf{L}_{i / k}=\left[\begin{array}{ccc}
\cos (n, x) & \cos (n, y) & 0 \\
\cos (s, x) & \cos (s, y) & 0 \\
0 & 0 & 1
\end{array}\right]
\end{gathered}
$$

Likewise, similar definitions can be made for element $j$, and the difference of velocity between element $i$ and $j$ can then be found as

$$
\dot{\boldsymbol{\varepsilon}}_{k}=\mathbf{L}_{i / k}\left(\mathbf{N}_{j / k} \dot{\mathbf{u}}_{j}-\mathbf{N}_{i / k} \dot{\mathbf{u}}_{i}\right)
$$

The following selection matrices $\mathbf{C}_{i}$ and $\mathbf{C}_{j}$ for elements $i$ and $j$ are introduced:

$$
\dot{\mathbf{u}}_{i(j)}=\mathbf{C}_{i(j)} \dot{\mathbf{U}}
$$

where $\dot{\mathbf{U}}^{T}=\left[\begin{array}{llll}\dot{\mathbf{u}}_{1}^{T} & \dot{\mathbf{u}}_{2}^{T} & \ldots & \dot{\mathbf{u}}_{m}^{T}\end{array}\right]_{(3 m \times 1)}=$ global velocity vector, where $m$ is the number of all the rigid elements. The selection matrix can be expressed as

$\mathbf{C}_{i(j)}=\left[\begin{array}{cccccccccc} & \multicolumn{1}{c}{3 i(j)-2} & 3 i(j)-1 & 3 i(j) & & & \\ 0 & \ldots & 0 & 0 & \hat{1} & \hat{0} & \hat{0} & 0 & \ldots & 0 \\ 0 & \ldots & 0 & 0 & 0 & 1 & 0 & 0 & \ldots & 0 \\ 0 & \ldots & 0 & 0 & 0 & 0 & 1 & 0 & \ldots & 0\end{array}\right]_{(3 \times 3 m)}$

Eq. (5) can then be reformulated as

$$
\dot{\boldsymbol{\varepsilon}}_{k}=\mathbf{B}_{k} \dot{\mathbf{U}}
$$

where

$$
\mathbf{B}_{k}=\mathbf{L}_{i / k}\left(\mathbf{N}_{j / k} \mathbf{C}_{j}-\mathbf{N}_{i / k} \mathbf{C}_{i}\right)
$$

As a result, the global discontinuous velocity vector Eq. (2) can be formulated as

$$
\dot{\boldsymbol{\varepsilon}}=\mathbf{B} \dot{\mathbf{U}}
$$

where $\mathbf{B}^{T}=\left[\begin{array}{llll}\mathbf{B}_{1}^{T} & \mathbf{B}_{2}^{T} & \ldots & \mathbf{B}_{n_{d}}^{T}\end{array}\right]_{\left(3 m \times 3 n_{d}\right)}=$ strain rate matrix. Eq. (10) represents the compatible condition between two adjacent rigid elements. This compatibility equation can be used to construct a numerical formulation for the consequent upper bound limit analysis.

\section{Equilibrium Equations for Rigid Element}

To apply the lower bound limit theorem, one needs to derive the equilibrium equation of rigid elements as well. Assume that the generalized stresses at the interface $k$ of a rigid element involve the shear force $V_{k}$, the normal force $N_{k}$, and the moment $M_{k}$, all applied at the center of the interface as shown in Fig. 2. They can be denoted in a vector form as

$$
\mathbf{Q}_{k}=\left[\begin{array}{lll}
N_{k} & V_{k} & M_{k}
\end{array}\right]^{T}, \quad k=1, \ldots, n_{d}
$$

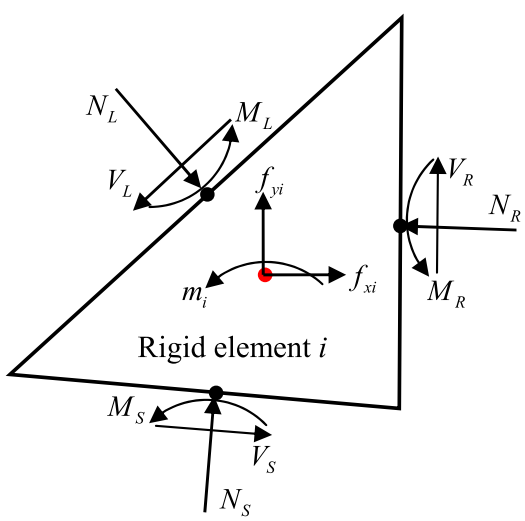

Fig. 2. Forces acting on a rigid finite element

The global stress vector can be written collectively as follows:

$$
\mathbf{Q}^{T}=\left[\begin{array}{llll}
\mathbf{Q}_{1}^{T} & \mathbf{Q}_{2}^{T} & \ldots & \mathbf{Q}_{n_{d}}^{T}
\end{array}\right]
$$

According to the principle of virtual work

$$
-\delta \dot{\boldsymbol{\varepsilon}}^{T} \mathbf{Q}-\delta \dot{\mathbf{U}}^{T} \cdot \lambda \mathbf{F}=0
$$

Substitution of Eq. (10) into Eq. (13) leads to

$$
-\delta \dot{\mathbf{U}}^{T} \mathbf{B}^{T} \mathbf{Q}-\lambda \delta \dot{\mathbf{U}}^{T} \mathbf{F}=0
$$

Considering $\delta \dot{\mathbf{U}} \neq 0$ in general cases gives the following global equilibrium equation:

$$
-\mathbf{B}^{T} \mathbf{Q}-\lambda \mathbf{F}=0
$$

where $\mathbf{F}^{T}=\left[\begin{array}{llll}\mathbf{f}_{1}^{T} & \mathbf{f}_{2}^{T} & \ldots & \mathbf{f}_{m}^{T}\end{array}\right]=$ global external force vector; and $\mathbf{f}_{i}=\left[\begin{array}{lll}f_{x i} & f_{y i} & m_{i}\end{array}\right]^{T}=i$ th component of $\mathbf{F}$. This equilibrium Eq. (13) is the same as the one derived by Qian and Zhang (1995) and Zhang (1999) using the principle of virtual work. It is also equivalent to the strong equilibrium equation proposed by Ferris and Tin-Loi (2001) and Orduna and Lourenco (2003).

\section{Yield Criteria and Flow Rules for Sliding and Rotation Mechanisms}

\section{Yield Criteria for Sliding and Rotation Mechanisms}

The kinematical admissible discontinuous velocity derived in the Equilibrium Equations for Rigid Element section can be separated into two failure modes as shown in Fig. 3, namely, relative sliding and rotation of an element with respect to its adjacent element. Accordingly, two different yield conditions are employed to govern the failures at the interface: a Mohr-Coulomb criterion for the sliding failure and an overturning failure criterion for the rotation failure.

\section{Sliding Mechanism between Two Rigid Elements}

Fig. 3(a) illustrates a translational sliding of element $j$ over element $i$. Mobilization of this translational mechanism may be described by the Mohr-Coulomb criterion, i.e.,

$$
|\tau|=c+\tan \varphi \sigma_{n}
$$



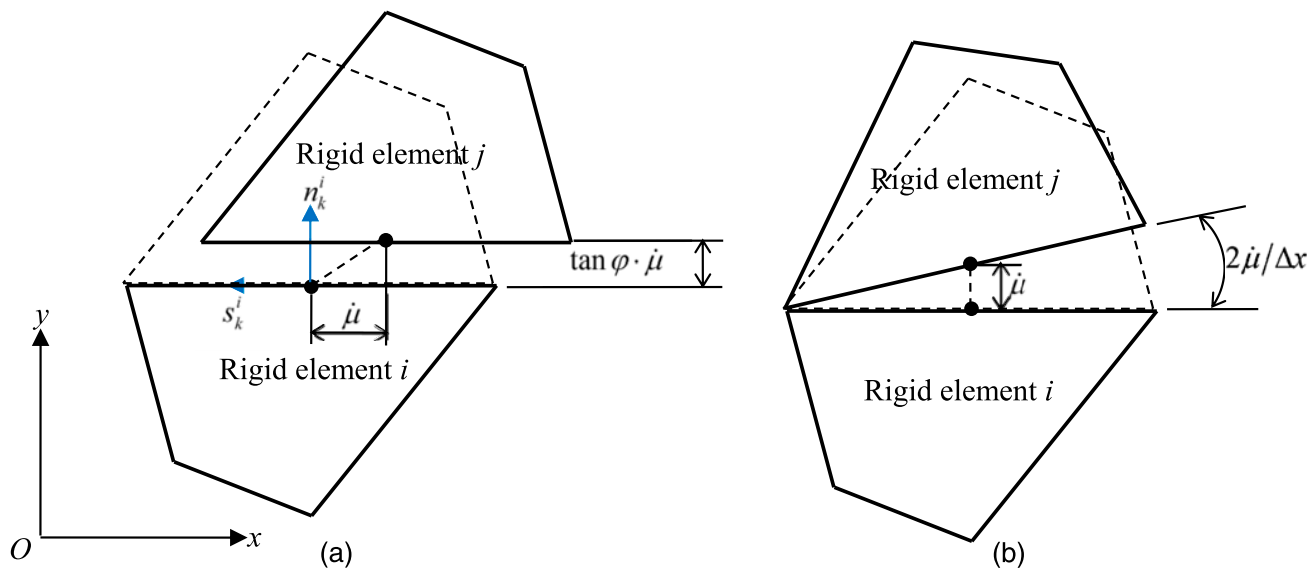

Fig. 3. Failure modes of interface between two rigid elements: (a) sliding; (b) rotation

Note that compression is taken to be positive here. Based on the generalized stress defined in Eq. (11), the above Mohr-Coulomb failure criterion may be recast to the following form:

$$
\left|V_{k}\right|-\tan \varphi N_{k}-c \Delta x \leq 0
$$

where $\Delta x=$ total length of interface. The normal and shear force acting on the $k$ th interface are defined as follows:

$$
N_{k}=\sigma \Delta x, \quad V_{k}=\tau \Delta x
$$

Note that the use of the absolute value of $V_{k}$ in Eq. (17) indicates that the shear direction is unrestricted.

\section{Rotational Failure Mechanism between Two Rigid Elements}

Element rotation has been ignored by previous RFEM-based limit analyses by Chen et al. (2003, 2004, 2005) and Zhang (1999), and the main purpose was to maintain the condition of no gap or overlap between neighboring elements. Although it appears to be reasonable for the translation-dominant type of slope failure, the assumption proves to be inadequate to describe cases where the rotation may be a contributing factor. In this paper, the authors will introduce an overturning failure criterion to govern both the kinematically and statically admissible fields in the limit analysis. The criterion is generalized based on the toppling failure criterion originally proposed by Goodman and Bray (1976). Consider a block on an inclined surface subjected to self-weight only (Fig. 4). Fig. 4 shows a state in which a rotation or toppling of the block is pending. The critical condition can be expressed as

$$
\frac{y}{\Delta x} \leq \cot \alpha
$$

where $y=$ height of the block; $\Delta x=$ width of the block; and $\alpha=$ inclined angle of the surface. By multiplying both sides of the inequality in Eq. (19) with the weight $W$ of the block, the following expression of the failure criterion is obtained:

$$
M_{k}-N_{k} \frac{\Delta x_{k}}{2} \leq 0
$$

where

$$
M_{k}=\frac{W \cdot \sin \alpha \cdot y}{2}, \quad N_{k}=W \cdot \cos \alpha
$$

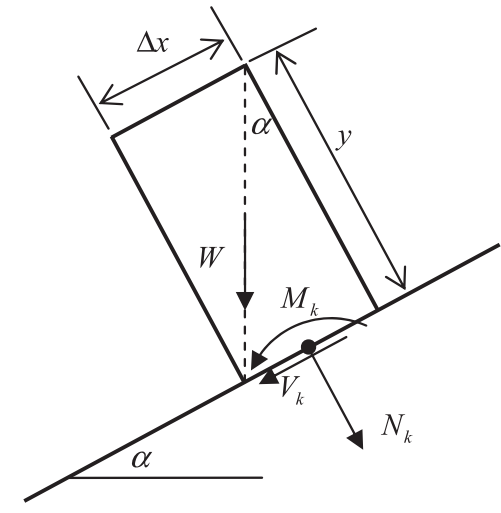

Fig. 4. Failure criterion for block toppling [adapted from Goodman and Bray (1976)]

Assuming that the moment $M_{k}$ at the $k$ th interface is an unrestricted variable gives the following overturning failure criterion:

$$
\left|M_{k}\right|-N_{k} \frac{\Delta x_{k}}{2} \leq 0
$$

To facilitate the subsequent numerical implementation, the sliding failure criterion in Eq. (17) and the overturning failure criterion in Eq. (20) for the $k$ th interface can be collectively expressed in the following matrix form:

$$
\boldsymbol{\varphi}_{k}=\boldsymbol{\Phi}_{k}^{T} \mathbf{Q}_{k}+\mathbf{a}_{k} \leq 0
$$

where

$$
\boldsymbol{\Phi}_{k}^{T}=\left[\begin{array}{ccc}
-\tan \varphi_{k} & 1 & 0 \\
-\tan \varphi_{k} & -1 & 0 \\
-\frac{\Delta x_{k}}{2} & 0 & 1 \\
-\frac{\Delta x_{k}}{2} & 0 & -1
\end{array}\right] ; \quad \mathbf{a}_{k}=\left[\begin{array}{c}
-c_{k} \Delta x_{k} \\
-c_{k} \Delta x_{k} \\
0 \\
0
\end{array}\right]
$$

where $\varphi_{k}$ and $c_{k}=$ friction angle and cohesion at the $k$ th interface, respectively. The failure criteria at all interfaces can then be assembled in the following matrix formulation: 


$$
\boldsymbol{\varphi}=\boldsymbol{\Phi}^{T} \mathbf{Q}+\mathbf{a} \leq 0
$$

where

$$
\begin{gathered}
\boldsymbol{\varphi}^{T}=\left[\begin{array}{cccc}
\boldsymbol{\varphi}_{1}^{T} & \boldsymbol{\varphi}_{2}^{T} & \ldots & \boldsymbol{\varphi}_{n_{d}}^{T}
\end{array}\right]_{\left(1 \times 4 n_{d}\right)} \\
\boldsymbol{\Phi}^{T}=\left[\begin{array}{cccc}
\boldsymbol{\Phi}_{1}^{T} & \mathbf{0} & \ldots & \mathbf{0} \\
\mathbf{0} & \boldsymbol{\Phi}_{2}^{T} & \ldots & \mathbf{0} \\
\vdots & \vdots & \ddots & \vdots \\
\mathbf{0} & \mathbf{0} & \mathbf{0} & \boldsymbol{\Phi}_{n_{d}}^{T}
\end{array}\right]_{\left(4 n_{d} \times 3 n_{d}\right)} \\
\mathbf{a}^{T}=\left[\begin{array}{llll}
\mathbf{a}_{1}^{T} & \mathbf{a}_{2}^{T} & \ldots & \mathbf{a}_{n_{d}}^{T}
\end{array}\right]
\end{gathered}
$$

where $n_{d}=$ number of interfaces in the discretized domain.

\section{Associated Flow Rule}

In conjunction with the previously given failure criteria, additional constraints on the kinematically admissible velocity field are needed by considering the associated flow rule. For interface sliding, the associated flow rule assumes that the tangential velocity change, $\dot{\mu}$, is accompanied by the separation velocity, $\tan \varphi \cdot \dot{\mu}$ [Fig. 3(a)]. As for the interface overturning, the associated flow rule requires that the normal velocity change $\dot{\mu}$ corresponds to an opening angle change of $2 \dot{\mu} / \Delta x$ [Fig. 3(b)]. In relation to the velocity vector in Eq. (1), these kinematic slip and rotation conditions can be expressed as $\dot{\boldsymbol{\varepsilon}}_{i}=-\dot{\mu}_{k} \cdot \partial \boldsymbol{\varphi}_{k} / \partial \mathbf{Q}_{i}$, where $\boldsymbol{\varphi}_{k}$ stands for the failure criterion corresponding to the $k$ th interface. Assembling all the associated flow equations for the interface gives

$$
\dot{\boldsymbol{\varepsilon}}_{k}=-\Phi_{k} \dot{\boldsymbol{\mu}}_{k}
$$

where $\dot{\boldsymbol{\mu}}_{k}=\left[\begin{array}{llll}\mu_{1 k} & \mu_{2 k} & \mu_{3 k} & \mu_{4 k}\end{array}\right]^{T}=$ vector of nonnegative plastic multipliers. By substituting Eq. (26) into Eq. (10), the compatibility condition can be reformulated as follows:

$$
\mathbf{B} \dot{\mathbf{U}}=-\Phi \dot{\boldsymbol{\mu}}
$$

With the previously given compatibility equation and equilibrium equation, the upper and lower bound limit analysis can be treated as a unified mathematical programming problem, which will be conducted in the Linear Programming for RFEM-Based Limit Analysis section.

\section{Linear Programming for RFEM-Based Limit Analysis}

As shown previously, the kinematically admissible velocity field has been separated into sliding and rotational modes, and the relative rotation between two rigid elements is governed by the toppling failure criterion in Eq. (22). Following the assumption of the associated flow rule, the relative rotation is further introduced into the governing equations of the kinematically and statically admissible fields in the limit analysis. In addition, the compatibility, equilibrium, and failure equations formulated previously are all in linear form. Taking this feature, the authors can then formulate the upper and lower bound theorems into a dual of two linear programming problems.

\section{Primal Problem of Linear Programming for Kinematic Approach}

The upper bound approach of limit theory states that any limit load multiplier $\lambda$ for a kinematically admissible velocity restricted by the compatibility equation and associated flow rule, i.e., Eq. (27), cannot be smaller than the plastic collapse multiplier $\lambda_{c}$, i.e., $\lambda \leq \lambda_{c}$. An upper bound limit analysis aims to find the minimum of the load multiplier $\lambda_{\min }$ to approximate $\lambda_{c}$. Using the associated flow rule in Eq. (26) in the virtual principle in Eq. (13) gives the following equation for the load multiplier:

$$
\dot{\boldsymbol{\mu}}^{T} \boldsymbol{\Phi}^{T} \mathbf{Q}-\lambda \dot{\mathbf{U}}^{T} \mathbf{F}=0
$$

Further substitution of the failure criteria in Eq. (25) into the previously revised virtual principle leads to

$$
\dot{\boldsymbol{\mu}}^{T}(\boldsymbol{\varphi}-\mathbf{a})-\lambda \dot{\mathbf{U}}^{T} \mathbf{F}=0
$$

By considering the complementary condition $\dot{\boldsymbol{\mu}}^{T} \boldsymbol{\varphi}=0$, the load multiplier can be formulated as follows:

$$
\lambda=-\dot{\boldsymbol{\mu}}^{T} \mathbf{a} \quad \text { when } \dot{\mathbf{U}}^{T} \mathbf{F}=1
$$

As a result, the authors can formulate the upper bound approach as the following linear programming problem:

$$
\min \lambda=-\dot{\boldsymbol{\mu}}^{T} \mathbf{a} \quad \text { such that } \dot{\mathbf{U}}^{T} \mathbf{F}=1, \quad \mathbf{B} \dot{\mathbf{U}}=-\boldsymbol{\Phi} \dot{\boldsymbol{\mu}}, \quad \dot{\boldsymbol{\mu}} \geq \mathbf{0}
$$

Note that boundary conditions in the upper bound approach constitute mainly prescribed velocities, for example, $\dot{\mathbf{U}}=\mathbf{0}$. Although they do not contribute to the real velocity field, the generalized primal variables in Eq. (31) will not include the quantities on the prescribed boundaries.

\section{Dual Problem for Static Approach}

In the lower bound approach of limit theory, any load multiplier $\lambda$ corresponding to a statically admissible stress field constrained by the equilibrium equation in Eq. (15) and the failure criteria Eq. (25) cannot be greater than the plastic collapse multiplier $\lambda_{c}$, i.e., $\lambda \geq \lambda_{c}$. The linear programming problem of a lower bound approach is to find the maximum of the load multiplier $\lambda_{\max }$. Alternatively, according to the dual theory of linear programming, the lower bound approach can be also formulated as the following dual of the primal problem of the corresponding upper bound approach:

$$
\max \lambda \quad \text { such that }-\mathbf{B}^{T} \mathbf{Q}-\lambda \mathbf{F}=\mathbf{0}, \quad \boldsymbol{\varphi}=\boldsymbol{\Phi}^{T} \mathbf{Q}+\mathbf{a} \leq 0
$$

The reactions on the boundary can be calculated by the compatibility matrix $\mathbf{B}$ for elements on the traction boundary and the stresses $\mathbf{Q}$, e.g., $\mathbf{F}_{b}=\mathbf{B}_{b}^{T} \mathbf{Q}$.

According to the duality theory of linear programming, the upper (primal) and the lower (dual) bound solutions can be found by solving the following optimal [Karush- Kuhn-Tucker (KKT)] conditions:

$$
\left[\begin{array}{cccc}
0 & \mathbf{0} & \mathbf{F}^{T} & \mathbf{0} \\
\mathbf{0} & \mathbf{0} & \mathbf{B} & \boldsymbol{\Phi} \\
-\mathbf{F} & -\mathbf{B}^{T} & \mathbf{0} & \mathbf{0} \\
\mathbf{0} & -\boldsymbol{\Phi}^{T} & \mathbf{0} & \mathbf{0}
\end{array}\right]\left[\begin{array}{c}
\lambda \\
\mathbf{Q} \\
\dot{\mathbf{U}} \\
\boldsymbol{\mu}
\end{array}\right]+\left[\begin{array}{c}
0 \\
\mathbf{0} \\
\mathbf{0} \\
-\boldsymbol{\varphi}
\end{array}\right]=\left[\begin{array}{c}
1 \\
\mathbf{0} \\
\mathbf{0} \\
-\mathbf{a}
\end{array}\right]
$$

where $\boldsymbol{\varphi}^{T} \dot{\boldsymbol{\mu}}=0 ; \dot{\boldsymbol{\mu}} \geq \mathbf{0}$; and $\boldsymbol{\varphi} \leq \mathbf{0}$. The authors employ the primaldual interior-point method (IPM) proposed by Andersen et al. (1996) 
and a package called MOSEK with its implementation to find the exact limit solution of the linear programming problem.

\section{Numerical Verifications and Application}

The performance of the numerical approaches formulated previously will be verified by two classical problems first and will be further applied to an inhomogeneous slope problem. All calculations were performed on a personal computer with an Intel Core 2 Quad CPU $2.83 \mathrm{GHz}$ processor.

\section{Failure of Homogeneous Slope with Log-Spiral Rotational Failure Mechanism}

The authors first take an example of the stability of a homogeneous cohesive-frictional soil slope under self-weight as shown in Fig. 5(a). Taking a rotational discontinuity mechanism for the rigid body above the $\log$-spiral toe-failure slip surface $B C$ shown in Fig. 5(a), Chen (1975) derived an upper bound stability factor, $N_{s}=\gamma H / c$. For a given slope with slope angle $\beta$ and soil frictional angle $\varphi$, an upper bound has been found by minimizing a function of the two angles $\theta_{h}$ and $\theta_{o}$ shown in Fig. 5(a), $f\left(\theta_{h}, \theta_{o}\right)$. The same problem will be investigated by our RFEM-based upper bound approach here. To make it consistent with Chen's (1975) study, consider the rigid body $A B C$ to be a single rigid element. As shown in Fig. 5(b), the log-spiral slip surface is approximated by a number of line segments that constitute the interface between the rotation element $A B C$ and rest of the domain. In this study, an upper bound solution for the stability factor is sought by optimizing the unit weight of the soil mass with a load multiplier $\lambda$ for a slope with fixed height and cohesion. As a result, finding the minimum of the stability factor is equivalent to computing the minimum of the load multiplier as follows:

$$
N_{s}=\frac{\lambda_{\min } \gamma H}{c}
$$

In doing so, the authors set two ranges of $\theta_{h}$ and $\theta_{o}$ and plot the contours of the stability factor. For example, when the inclined angle of slope $\beta=45^{\circ}$, Fig. 6 shows the contours of the stability factor with four different frictional angles $\varphi=5,10,15$, and $20^{\circ}$. An adequate number of line segments for the interface is also required to gain good convergence of the stability factor, as shown in Fig. 7. Table 1 lists the obtained stability factors and the corresponding optimal angle $\theta_{h}$ and $\theta_{o}$ for different cases of slope angle and frictional angle. Our numerical results appear to be quite consistent with those obtained by Chen (1975).

\section{Planar Translational Failure with Tension Crack}

The authors wish to use two more examples in this section to highlight the importance of considering the rotational failure mechanism. In the first example, the authors consider only the sliding failure mechanism, whereas in the second example, they compare results obtained by considering the rotational failure mode with those without it.

\section{Dry Slope}

First, consider the same homogeneous slope as in the Failure of Homogeneous Slope with Log-Spiral Rotational Failure Mechanism section but with a tensor crack as shown in Fig. 8. In this case, the authors consider a planar translational failure mechanism. The example is similar to the plane failure of rock slopes analyzed by Hoek and Bray (1981). According to Hoek and Bray (1981), the potential failure plane should satisfy $\psi_{f}>\psi_{p}>\varphi$, where the three angles are shown in Fig. 8 .

Based on the LEM, the following general expression for the factor of safety can be obtained (Hoek and Bray 1981):

$$
F_{s}=\frac{2 c(1-z / H)}{\left\{\left[1-(z / H)^{2}\right] \cot \psi_{p}-\cot \psi_{f}\right\} \sin ^{2} \psi_{p}}+\cot \psi_{p} \tan \varphi
$$

The minimum factor of safety of the slope is obtained at a critical crack length, $z_{c}=\left(1-\sqrt{\cot \psi_{f} \tan \psi_{p}}\right) H$. Hoek and Bray (1981) have further defined the following dimensionless normal stress ratio:

$$
\frac{\sigma}{\gamma H}=\frac{\left\{\left[1-\left(z_{c} / H\right)^{2}\right] \cot \psi_{p}-\cot \psi_{f}\right\} \sin \psi_{p} \cos \psi_{p}}{2\left(1-z_{c} / H\right)}
$$

Note that in the original expression provided by Hoek and Bray (1981) the term $\cos \psi_{p}$ was inadvertently left out.

The authors have verified the problem using the RFEM-based limit analysis proposed in this paper. They treat the sliding block $A B C D$ and fixed-base block $A D E$ as two rigid elements. Although there is no obvious driving force that may lead to rotational failure for this example, the rotational failure mechanism in this method is naturally disabled. The authors employ the method of reducing strength to find the factor of safety for this problem, by following the common definition of the factor of safety

$$
F_{s}=\frac{c^{\prime}}{c_{m}^{\prime}}=\frac{\tan \varphi^{\prime}}{\tan \varphi_{m}^{\prime}}
$$

where $c^{\prime}$ and $\varphi^{\prime}=$ effective cohesion and internal frictional angle, respectively; and $c_{m}^{\prime}$ and $\varphi_{m}^{\prime}=$ reduced (mobilized) cohesion and frictional angle, respectively.

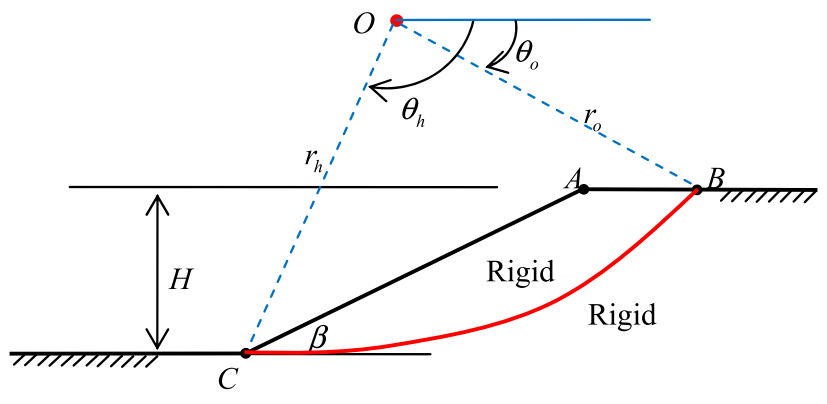

(a)

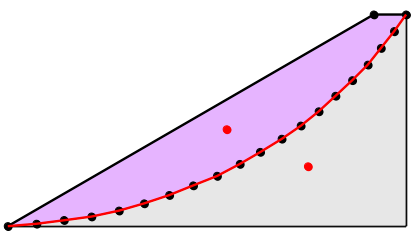

(b)

Fig. 5. Homogenous soil slope: (a) failure mechanism with a log-spiral slip surface passing the toe; (b) model of limit analysis based on RFEM 


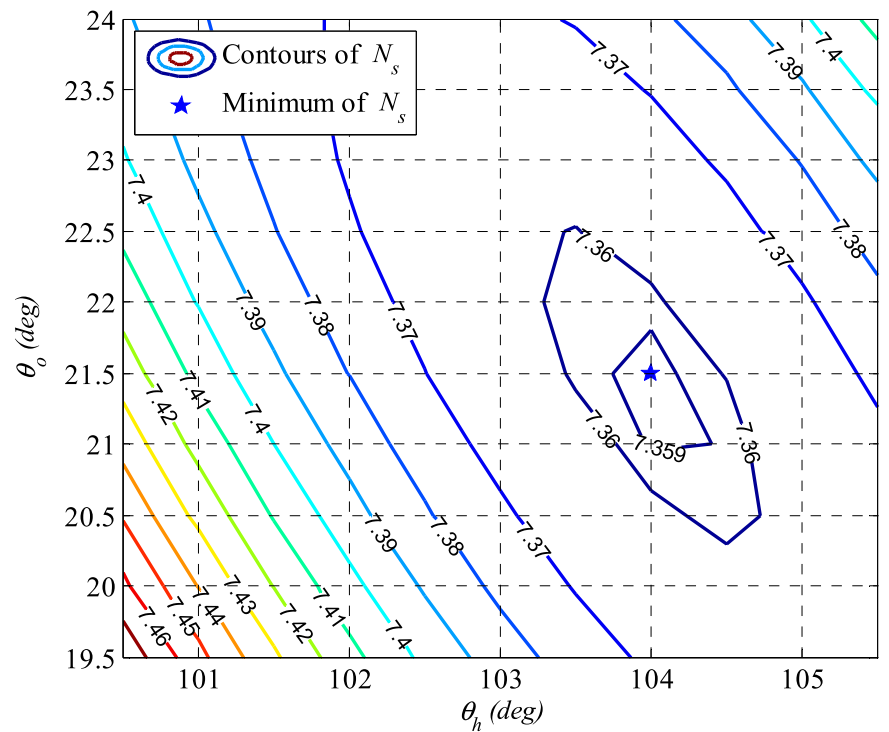

(a)

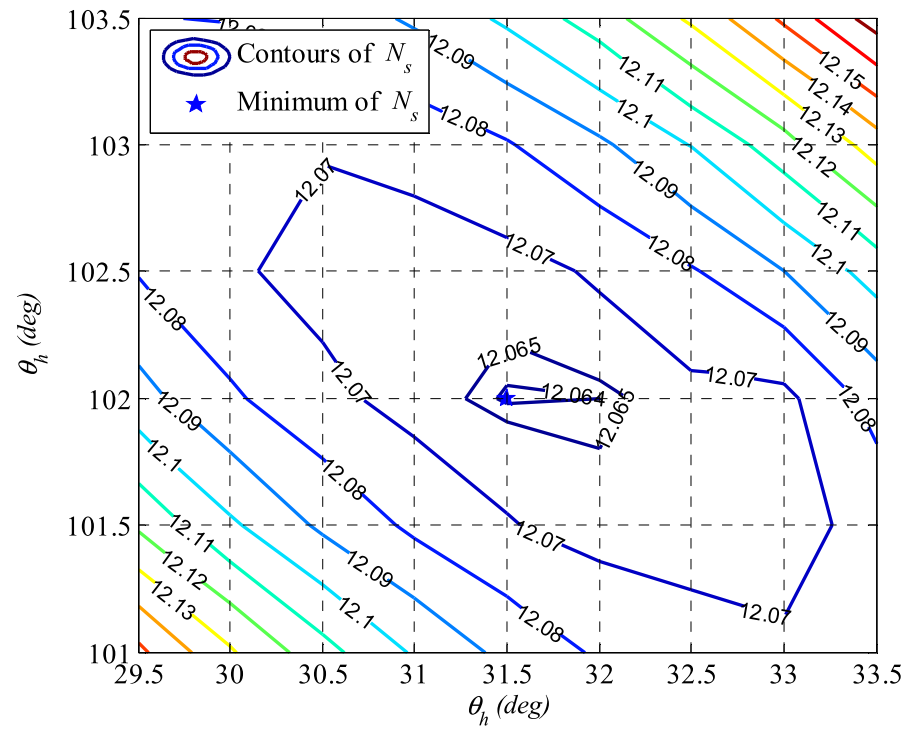

(c)

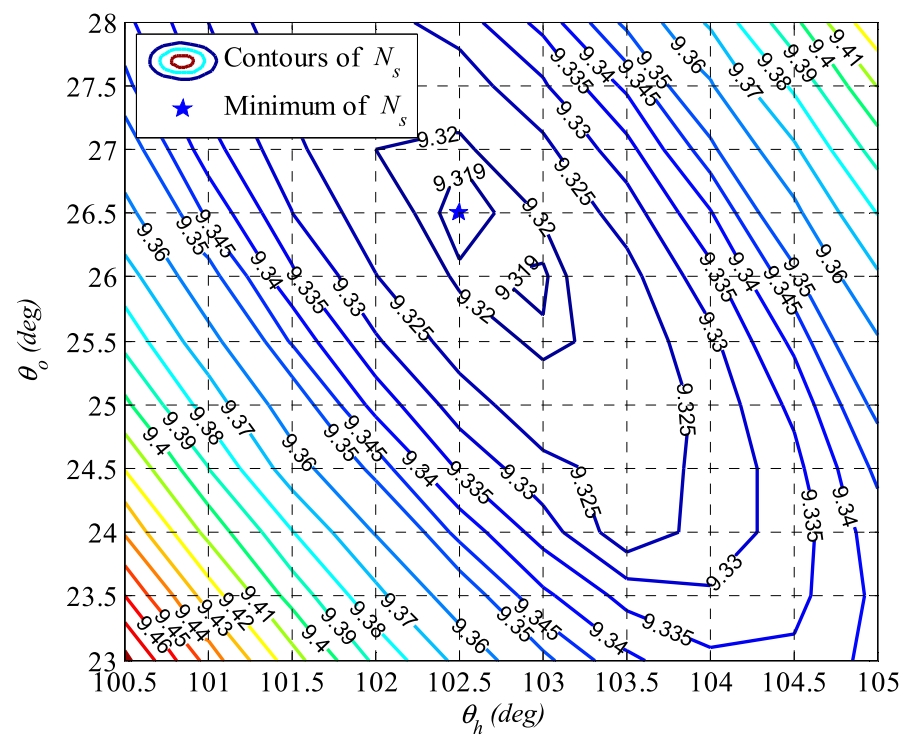

(b)

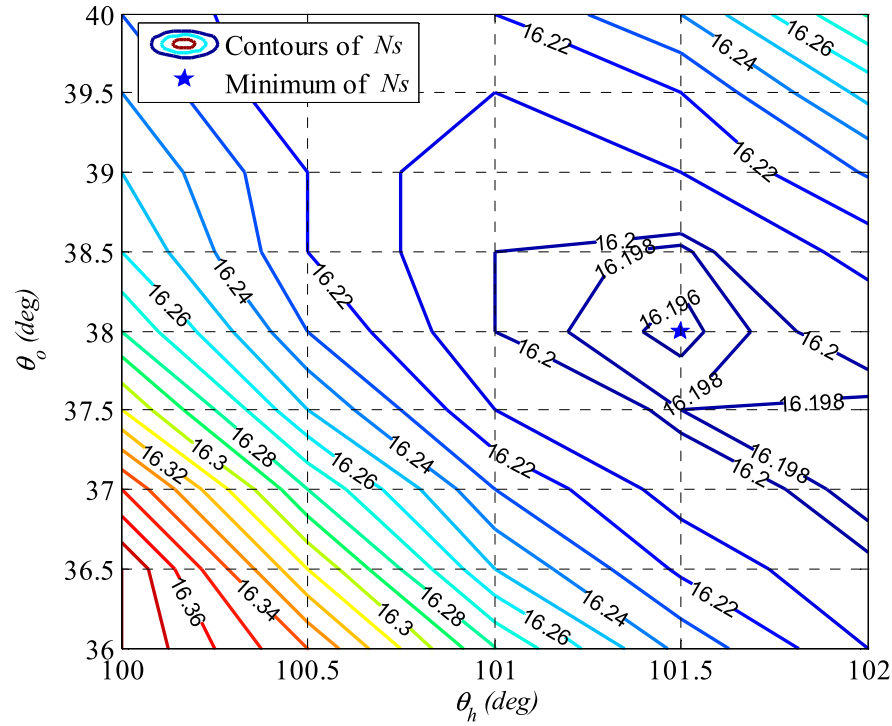

(d)

Fig. 6. Contours of stability factor of slope with inclined angle $\beta=45^{\circ}$ and different friction angles: (a) $\varphi=5^{\circ} ;$ (b) $\varphi=10^{\circ} ;\left(\right.$ c) $\varphi=15^{\circ} ;(d) \varphi=20^{\circ}$

The factor of safety $F_{s}$ is computed in conjunction with optimization of the limit load multiplier $\lambda$ according to Eq. (33). Specifically, an initial value $F_{s}$ (usually a small one) is chosen first to calculate the reduced cohesion and frictional angle $c_{m}^{\prime}$ and $\varphi_{m}^{\prime}$ according to Eq. (37). These two strength parameters are substituted into Eq. (24) to obtain an optimal limit load multiplier $\lambda$ using the proposed method. The process is repeated by gradually increasing the value of the factor of safety from its initial value. Consequently, a series of limit load multipliers in conjunction with the corresponding factor of safety can be obtained, which can be plotted in a figure like Fig. 9. The ultimate factor of safety for the problem corresponds to the value at the intersection of the curve with the horizontal line $\lambda=1$. For instance, for the case of a slope with a slope face $\psi_{f}=60^{\circ}$ and a failure plane $\psi_{p}=30^{\circ}$, a frictional angle $\varphi=20^{\circ}$ and cohesion $c^{\prime}=30 \mathrm{kN} / \mathrm{m}^{2}$, the factor of safety is determined to be $F_{s}=1.5411$ (marked as a star in Fig. 9) according to this procedure. Note that this method shares a certain similarity with the critical acceleration concept proposed by Sarma (1973).
Fig. 10 further presents the variation of normal stresses and the factor of safety with the slope and failure plane angles obtained by the present method in comparison with results obtained by the LEM from Hoek and Bray (1981). As is seen, when only the sliding failure mechanism is considered, the present method produces results identical to those of Hoek and Bray's (1981) LEM.

\section{Wet Slope}

This example involves the same geometry as the last one but differs from the last one in that there is a underground water table because of rainfall and the crack is filled with pore water to some height as well (as shown in Fig. 11). The authors further assume that the rest base is impermeable and that the water presenting in the crack as well as along the base $A D$ yields a linear pressure distribution as shown in Fig. 11. The height of water in the crack is assumed to be $z_{w}$, and $\gamma_{w}$ is the unit weight of water. The same problem was considered by Hoek and Bray (1981) using the LEM, and the factor of safety obtained therein is 


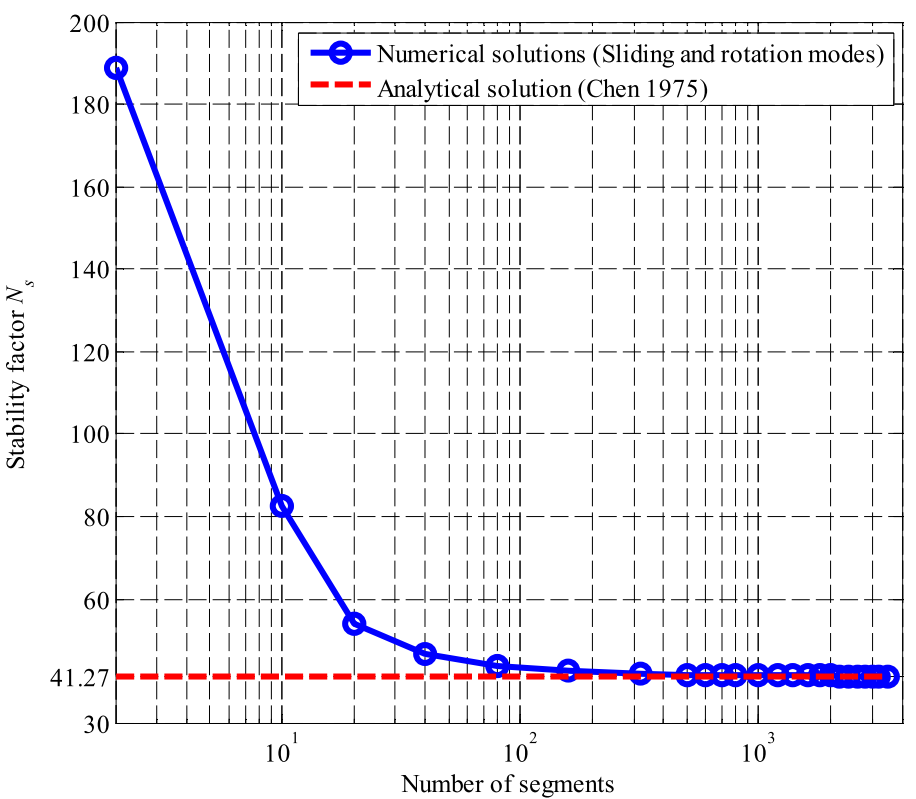

(a)

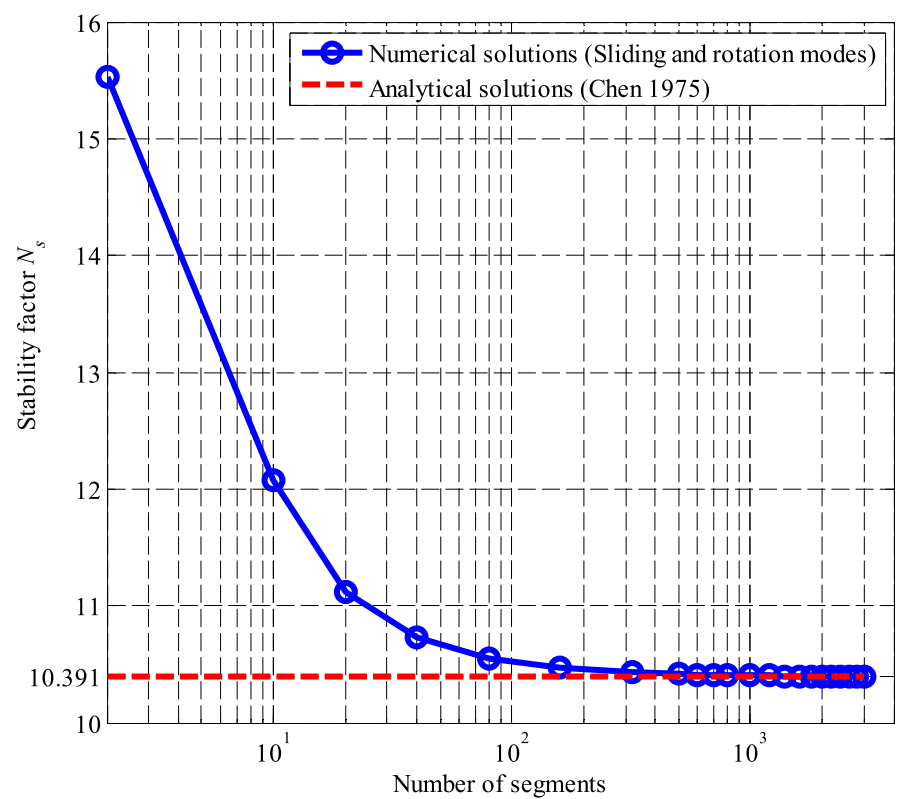

(b)

Fig. 7. Convergence of the upper bound stability factor by the current RFEM-based limit analysis with number of line segments for the interface: (a) $\beta=30^{\circ}, \varphi=20^{\circ}$; (b) $\beta=60^{\circ}, \varphi=20^{\circ}$

Table 1. Comparison of Stability Factors Obtained by the Analytical Method (Chen 1975) and the Present RFEM-Based Numerical Method

\begin{tabular}{|c|c|c|c|c|c|}
\hline \multirow{2}{*}{$\begin{array}{l}\text { Friction } \\
\text { angle } \varphi \\
\text { (degrees) }\end{array}$} & \multicolumn{5}{|c|}{ Slope angle $\beta$ (degrees) } \\
\hline & 90 & 75 & 60 & 45 & 30 \\
\hline \multicolumn{6}{|l|}{5} \\
\hline Chen's (1975) method & 4.1899 & 5.141 & 6.158 & 7.352 & 9.139 \\
\hline Present method & 4.1904 & 5.142 & 6.161 & 7.358 & 9.155 \\
\hline$\theta_{o}$ (degrees) & 31.0 & 26.5 & 21.5 & 21.5 & 25.5 \\
\hline$\theta_{h}$ (degrees) & 58.5 & 74.0 & 90.0 & 104.0 & 116.5 \\
\hline $\begin{array}{l}\text { Relative errors } \\
\text { (percentage) }\end{array}$ & 0.01 & 0.02 & 0.05 & 0.09 & 0.17 \\
\hline \multicolumn{6}{|l|}{10} \\
\hline Chen's (1975) method & 4.5829 & 5.800 & 7.258 & 9.310 & 13.502 \\
\hline Present method & 4.5833 & 5.801 & 7.262 & 9.318 & 13.523 \\
\hline$\theta_{o}$ (degrees) & 33.5 & 29.0 & 25.5 & 26.5 & 33.0 \\
\hline$\theta_{h}$ (degrees) & 60.0 & 75.0 & 89.5 & 102.5 & 113.5 \\
\hline $\begin{array}{l}\text { Relative errors } \\
\text { (percentage) }\end{array}$ & 0.01 & 0.02 & 0.05 & 0.09 & 0.16 \\
\hline \multicolumn{6}{|l|}{15} \\
\hline Chen's (1975) method & 5.0180 & 6.567 & 8.629 & 12.053 & 21.838 \\
\hline Present method & 5.0185 & 6.569 & 8.632 & 12.064 & 21.870 \\
\hline$\theta_{o}$ (degrees) & 31.0 & 31.5 & 29.0 & 31.5 & 46.0 \\
\hline$\theta_{h}$ (degrees) & 58.5 & 76.5 & 90.0 & 102.0 & 108.5 \\
\hline $\begin{array}{l}\text { Relative errors } \\
\text { (percentage) }\end{array}$ & 0.01 & 0.03 & 0.05 & 0.09 & 0.15 \\
\hline \multicolumn{6}{|l|}{20} \\
\hline Chen's (1975) method & 5.505 & 7.477 & 10.391 & 16.162 & 41.267 \\
\hline Present method & 5.506 & 7.480 & 10.395 & 16.174 & 41.321 \\
\hline$\theta_{o}$ (degrees) & 39.0 & 34.0 & 33.5 & 38 & 53.0 \\
\hline$\theta_{h}$ (degrees) & 63.5 & 78.0 & 90.5 & 101.5 & 108.0 \\
\hline $\begin{array}{l}\text { Relative errors } \\
\text { (percentage) }\end{array}$ & 0.01 & 0.03 & 0.04 & 0.08 & 0.13 \\
\hline
\end{tabular}

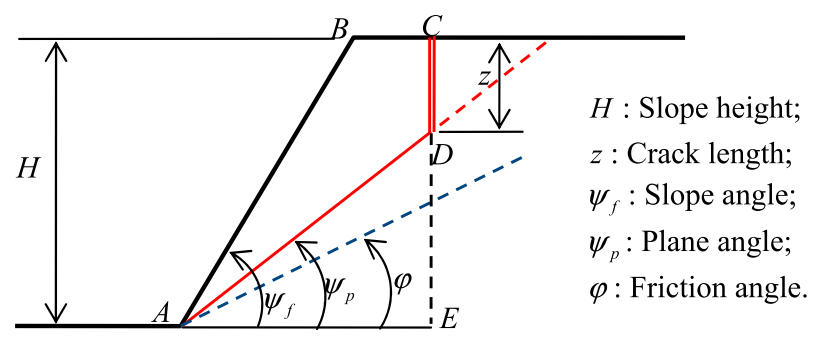

Fig. 8. Geometry of a dry slope with a tension crack in upper slope surface

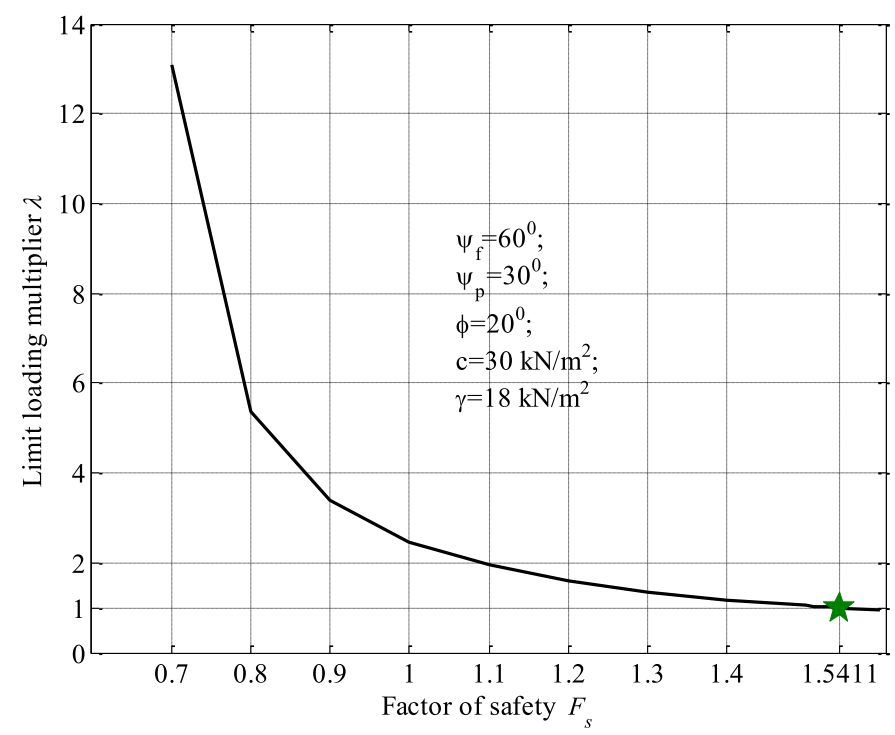

Fig. 9. Determination of the factor of safety by optimizing limit loading multiplier 


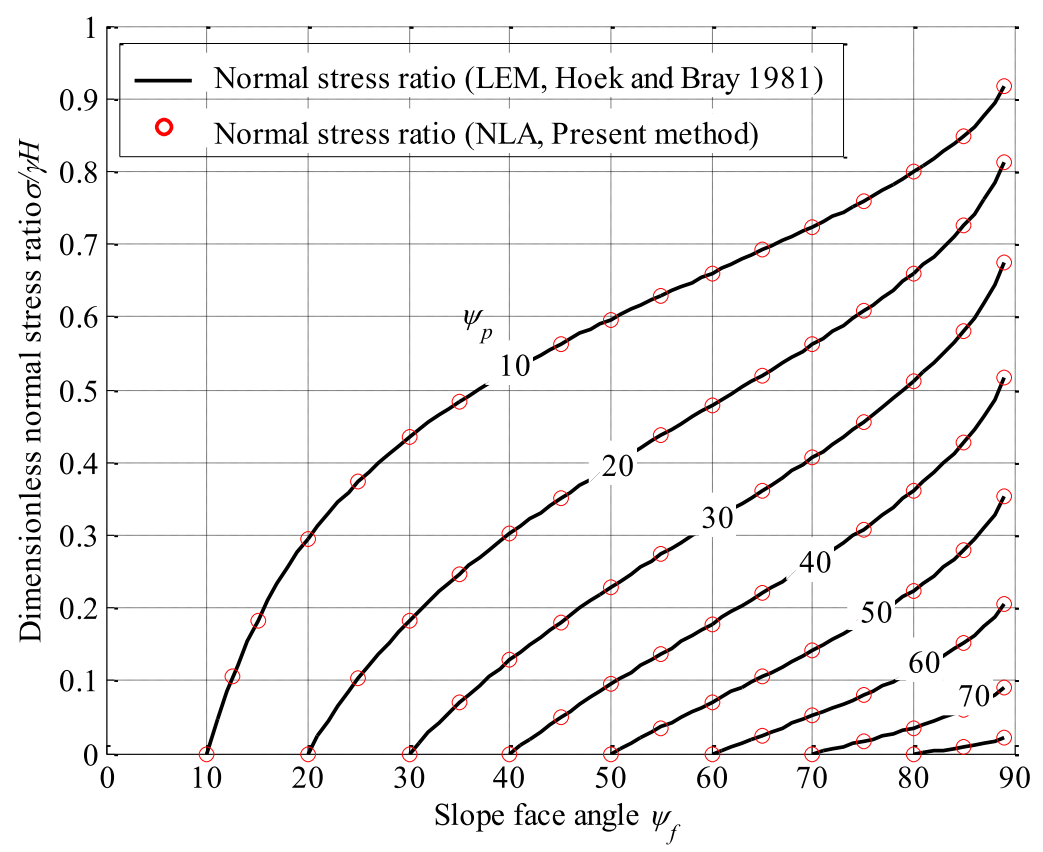

(a)

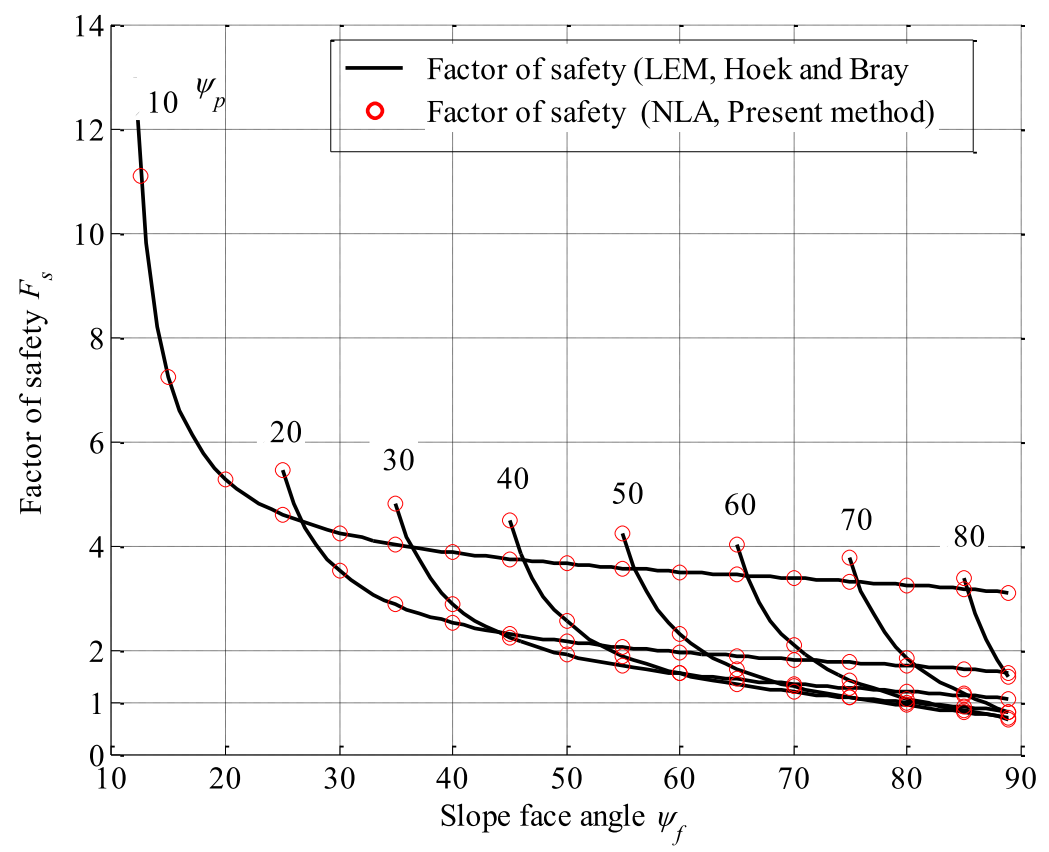

(b)

Fig. 10. Comparison between the LEM and the present method: (a) dimensionless normal stress ratio; (b) factor of safety

$F_{s}=\frac{(2 c / \gamma H) \cdot P+\left[Q \cdot \cot \psi_{p}-\left(\gamma_{w} z_{w} / \gamma H\right)\left(P+z_{w} \sin \psi_{p} / H\right)\right] \cdot \tan \varphi}{Q+\left(\gamma_{w} z_{w}^{2} / \gamma H^{2}\right) \sin \psi_{p} \cdot \cot \psi_{p}}$

where

$$
\begin{aligned}
& P=(1-z / H) / \sin \psi_{p} \\
& Q=\left\{\left[1-(z / H)^{2}\right] \cot \psi_{p}-\cot \psi_{f}\right\} \sin \psi_{p}
\end{aligned}
$$

It is noted, however, that in deriving Eq. (38) Hoek and Bray (1981) assumed that the uplift force attributable to water pressure on the sliding plane $A D$ and the driving force attributable to water pressure in the tension crack all act through the centroid of the sliding mass $A B C D$. The factor of safety defined in Eq. (39) predicts sliding failure of the slope along $A D$ only according to

$$
F_{s-\text { sliding }}=\frac{\sum \text { Forces }_{\text {resisting }}}{\sum \text { Forces }_{\text {driving }}}
$$




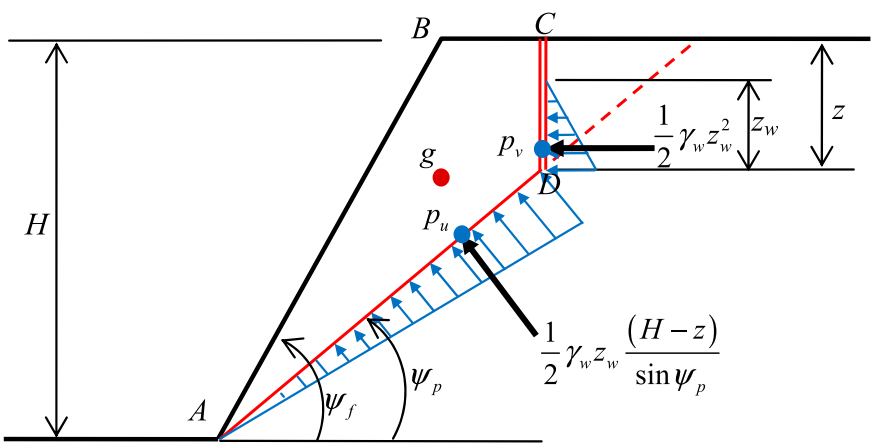

Fig. 11. Geometry of a wet rock slope with a tension crack in upper slope surface

Indeed, in the presence of pore water pressure along $A D$ and in the crack, it is also possible that overturning failure around $A$ may happen, for which another factor of safety characterizing the toppling or rotation failure of the slope needs to be defined as follows:

$$
F_{s-\text { toppling }}=\frac{\sum \text { Moments }_{\text {resisting }}}{\sum \text { Moments }_{\text {driving }}}
$$

The RFEM-based limit analysis method has been used to calculate the factor of safety for this slope where both the sliding and rotational failure mechanisms are considered. Specifically, the two

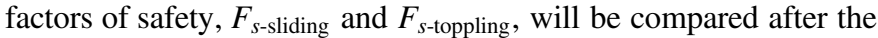
computation. If $F_{s \text {-sliding }}<F_{s \text {-toppling, }}$, the slope failure is controlled by sliding. Otherwise, the toppling failure mode will dominate. The smaller one between the two will be adopted as the final factor of safety for the slope. Fig. 12 presents the obtained results with the increase of the water level in the crack at different slope angles. The sliding failure results predicted by the LEM (Hoek and Bray 1981) are also given for comparison. It is evident from the figure that when the slope angles are low, e.g., in the cases shown in Fig. 12(a), the increase of the water level in the crack does not affect the failure mechanism, which is still sliding in nature. However, when the slope angles become higher, the impact of the water level on the failure mode becomes evident. Notably from Figs. 12(b-d), when the water level in the crack is low, the failure of the slope is still dominated by the sliding mode, and the authors' predictions of the factor of safety are identical with those of the LEM. As the water level is increased, however, the rotational failure mode may be mobilized before the sliding failure. Consequently, the LEM predictions may lead to a greater factor of safety than the authors' method, and in some cases, the difference may exceed $90 \%$ (such as in the case $\psi_{f}=80^{\circ}$, $\psi_{p}=70^{\circ}$, and $z_{w} / z=0.6$ ). More specifically, at the same slope angle, the higher the failure plane angle is, the lower the water level that is needed to mobilize a toppling failure for the slope. It is evident from the example that the consideration of the rotational/toppling failure mode in conjunction with the sliding failure mode may lead to a safer design for the slope than considering the latter only.

\section{Inhomogeneous Soil Slope with Weaker Layer}

The present method will be further applied to evaluate the stability of an inhomogeneous soil slope previously treated by Kim et al. (2002). Presented in Fig. 13 are the geometry of the slope and the profile of the soil layers. The shear strength parameters and unit weight of each layer are also summarized in Table 2. Apparently, Soil 2 serves as a relatively weak layer in between Soil 1 and Soil 3.

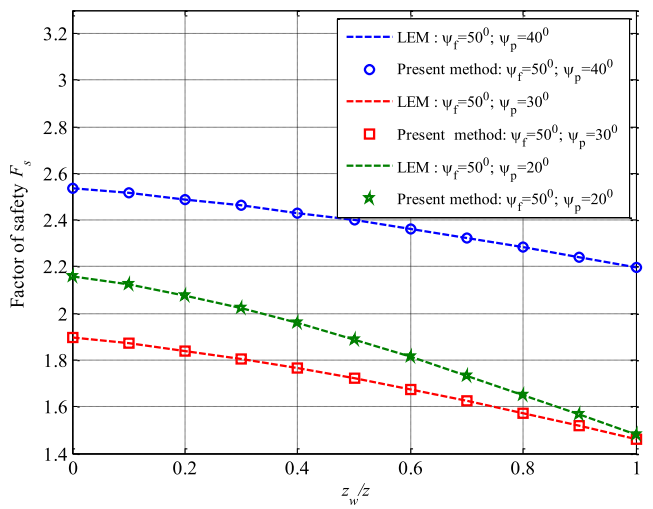

(a)

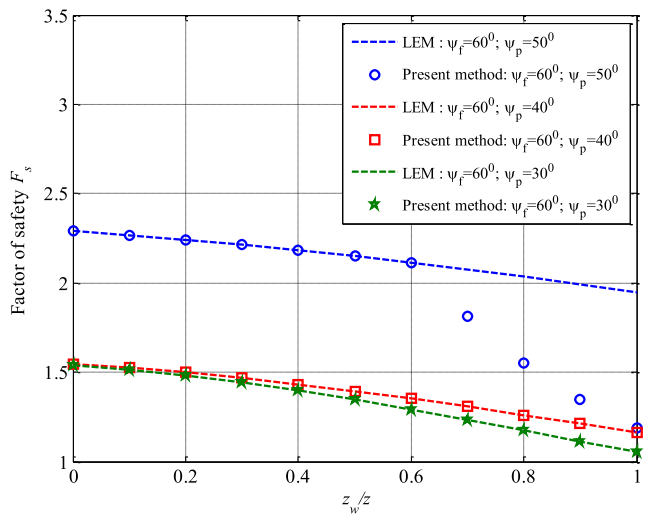

(b)

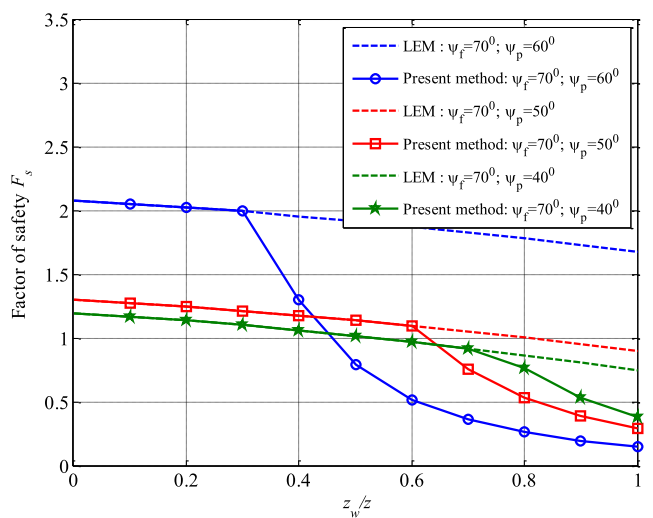

(c)

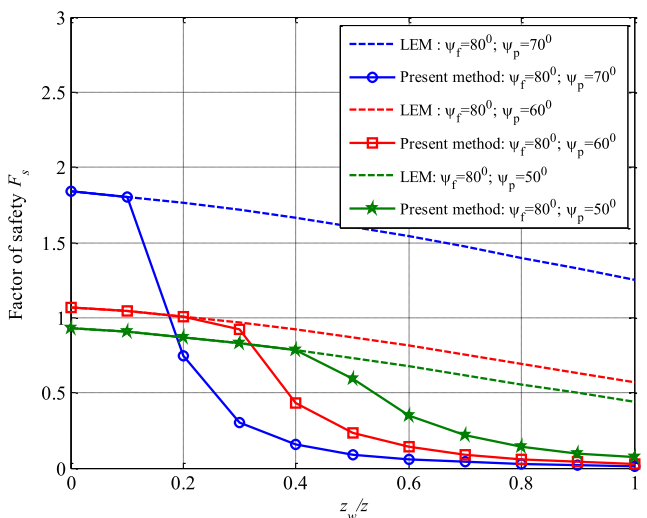

(d)

Fig. 12. Comparison of the factors of safety between the present method and LEM: (a) $\psi_{f}=50^{\circ}$; (b) $\psi_{f}=60^{\circ}$; (c) $\psi_{f}=70^{\circ}$; (d) $\psi_{f}=80^{\circ}$ 


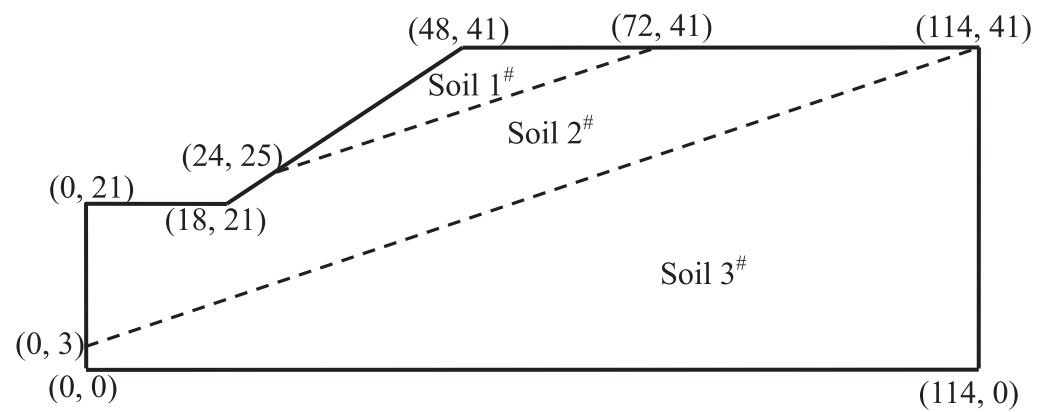

Fig. 13. Inhomogeneous slope with three layers of soil [adapted from Kim et al. (2002)]

Table 2. Shear Strength Parameters and Unit Weights of Soils

\begin{tabular}{lccc}
\hline Soils & $c\left(\mathrm{kN} / \mathrm{m}^{2}\right)$ & $\varphi$ (degrees) & $\gamma\left(\mathrm{kN} / \mathrm{m}^{3}\right)$ \\
\hline 1 & 29.4 & 12.0 & 18.8 \\
2 & 9.8 & 5.0 & 18.8 \\
3 & 294 & 40.0 & 18.8 \\
\hline
\end{tabular}

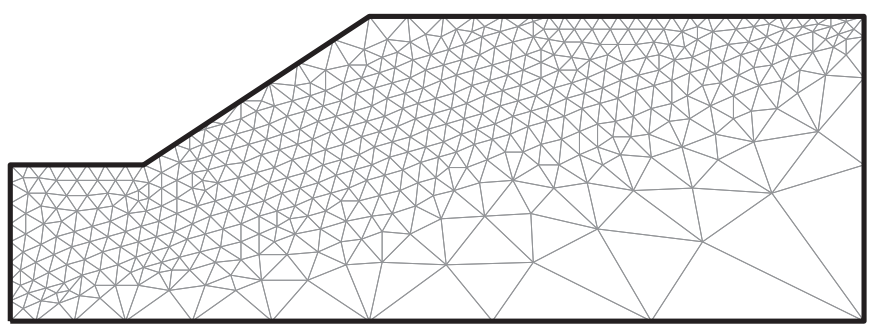

Fig. 14. Typical rigid finite-element mesh for the inhomogeneous slope

The domain is discretized by an unstructured triangle mesh as shown in Fig. 14. The factor of safety of the problem is then solved by the proposed method with the same procedure as described in the Tension Crack section. Fig. 15 shows the consistency of the results with the refinement of the mesh. When the mesh is fine enough, a consistent factor of safety is obtained by the proposed method for the cases considering and not considering the rotational failure mode. As is summarized in Table 3, in particular, the factor of safety in consideration for both sliding and rotation failure modes is found between the upper bound and lower bound obtained by Kim et al. (2002) and is greater than that obtained by Spencer's method (Greco 1996). If only sliding failure is considered, however, the obtained factor of safety is significantly larger than all the cases mentioned previously. For example, it is around $30 \%$ greater than the value obtained by considering both failure mechanisms. Fig. 16 further presents a comparison of the kinematically admissible velocity fields obtained for the two cases: (a) by considering sliding failure only and (b) by considering both sliding and rotational failure modes. As is shown in Fig. 16, rotational zones can be clearly observed in the second cases at the crest part of the failure zone as compared with the largely translational failure in the first case. Finally, though not presented here, the primal-dual interior-point method also proves to be more efficient than other approaches in solving the problem, such as the Simplex method (Dantzig et al. 1955), especially when a fine mesh is used.

\section{Conclusions}

Based on the RFEM, a general formulation of upper and lower bound limit analysis has been presented. The upper and lower bound

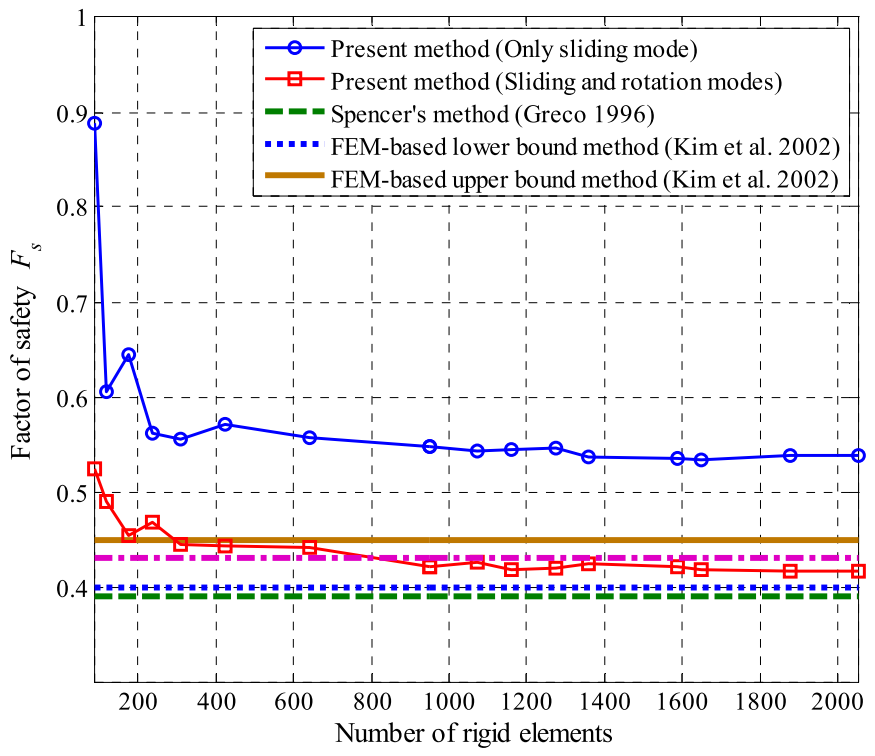

Fig. 15. Relationship between factor of safety and number of rigid elements

Table 3. Comparison of Factor of Safety by Various Methods

\begin{tabular}{lll}
\hline Authors & \multicolumn{1}{c}{ Methods } & $F_{s}$ \\
\hline Present method & Sliding & 0.547 \\
Present method & Sliding and rotation & 0.421 \\
Greco (1996) & Spencer's method (Monte Carlo method) & 0.39 \\
Greco (1996) & Spencer's method (pattern search method) & 0.39 \\
Kim et al. (2002) & Bishop's method & 0.43 \\
Kim et al. (2002) & FEM-based lower bound method & 0.40 \\
Kim et al. (2002) & FEM-based upper bound method & 0.45 \\
\hline
\end{tabular}

solution is expressed as primal and dual linear programming problems that can be solved by an efficient and robust primal-dual interior-point method. The novelty in the approach is the consideration of both sliding and rotational failure mechanisms, which is general in form and covers the traditional LEM as a special case. Numerical examples demonstrate that the rotational failure mode may be mobilized before the sliding failure mode in some cases, which may result in a lower factor of safety for a slope problem. The proposed method may hence provide an efficient and safe way for practical slope design. Future study will focus on the application of the method to more complex problems involving block toppling and flexural toppling, which are commonly observed in rock slopes. 


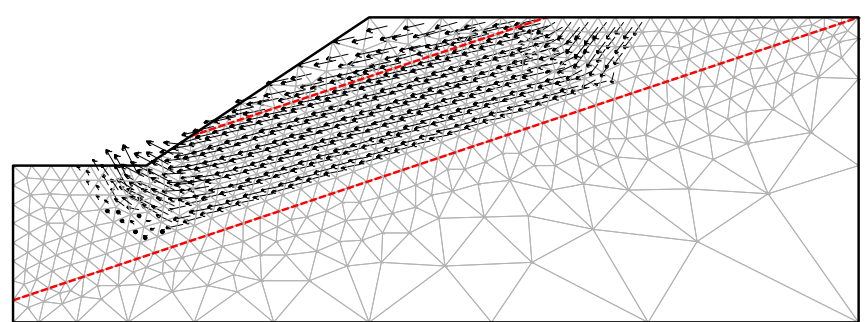

(a)

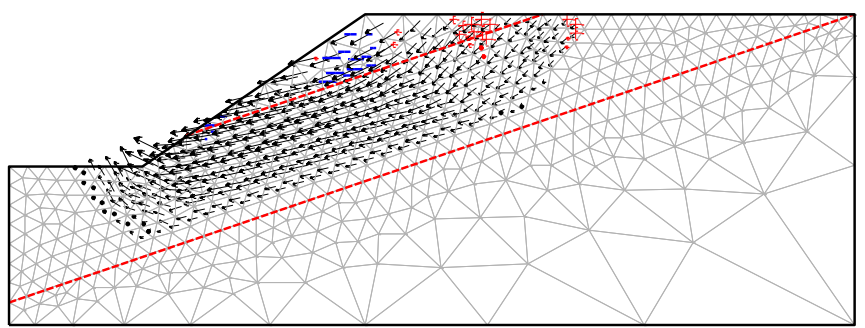

(b)

Fig. 16. Kinematically admissible velocity fields obtained by (a) considering sliding failure mode only; (b) considering both sliding and rotational failure mechanisms (the plus sign denotes anticlockwise rotation, and the minus sign denotes clockwise rotation, with the magnitude being proportional to the size of the sign)

\section{Acknowledgments}

The work was supported by the Research Grants Council of Hong Kong (under grant no. 623609) and HKUST Postdoctoral Fellowship Matching Fund. The first author also appreciates that MOSEK ApS has kindly provided a free academic license of MOSEK for the study.

\section{References}

Ahmed, A., Ugai, K., and Yang, Q. Q. (2012). “Assessment of 3D slope stability analysis methods based on 3D simplified Janbu and Hovland methods." Int. J. Geomech., 12(2), 81-89.

Amini, M., Majdi, A., and Aydan, Ö. (2009). "Stability analysis and the stabilization of flexural toppling failure." Rock Mech. Rock Eng., 42(5), 751-782.

Andersen, E. D., Gondzio, J., Meszaros, C., and Xu, X. (1996). "Implementation of interior point methods for large scale linear programming." Interior-point methods of mathematical programming, T. Terlaky, ed., Kluwer, Dordrecht, Netherlands, 189-252.

Chen, J., Yin, J. H., and Lee, C. F. (2003). "Upper bound limit analysis of slope stability using rigid finite elements and nonlinear programming." Can. Geotech. J., 40(4), 742-752.

Chen, J., Yin, J. H., and Lee, C. F. (2004). "A rigid finite element method for upper bound limit analysis of soil slopes subjected to pore water pressure." J. Eng. Mech., 130(8), 886-893.

Chen, J., Yin, J. H., and Lee, C. F. (2005). "A three-dimensional upperbound approach to slope stability analysis based on RFEM." Geotechnique, 55(7), 549-556.

Chen, W. F. (1975). Limit analysis and soil plasticity, Elsevier, Amsterdam, Netherlands.

Chen, Z., Wang, X., Haberfield, C., Yin, J. H., and Wang, Y. (2001a). "A three-dimensional slope stability analysis method using the upper bound theorem: Part I: Theory and methods." Int. J. Rock Mech. Min. Sci., 38(3), 369-378.

Chen, Z., Wang, X., Wang, Y., Yin, J. H., and Haberfield, C. (2001b). "A three-dimensional slope stability analysis method using the upper bound theorem: Part II: Numerical approaches, applications and extensions." Int. J. Rock Mech. Min. Sci., 38(3), 379-397.

Cruden, D. M. (1989). "Limits to common toppling." Can. Geotech. J., 26(4), 737-742.

Dantzig, G. B., Orden, A., and Philip, W. (1955). "The generalized simplex method for minimizing a linear form under linear inequality restraints." Pac. J. Math., 5(2), 183-195.

Donald, I. B., and Chen, Z. (1997). "Slope stability analysis by the upper bound approach: Fundamentals and methods." Can. Geotech. J., 34(6), 853-862.

Drucker, D. C., and Prager, W. (1952). "Soil mechanics and plastic analysis or limit design." Q. Appl. Math., 10(2), 157-165.

Ferris, M. C., and Tin-Loi, F. (2001). "Limit analysis of frictional block assemblies as a mathematical program with complementarity constraints." Int. J. Mech. Sci., 43(1), 209-224.

Goodman, R. E., and Bray, J. W. (1976). "Toppling of rock slopes." Proc., Specialty Conf. on Rock Engineering for Foundations and Slopes, ASCE, New York, 269-321.

Greco, V. R. (1996). "Efficient Monte Carlo technique for locating critical slip surface." J. Geotech. Engrg., 122(7), 517-525.

Hoek, E., and Bray, J. W. (1981). Rock slope engineering, 3rd Ed., Institute of Mining and Metallurgy, London.

Kawai, T. (1978). "New discrete models and their application to seismic response analysis of structures." Nucl. Eng. Des., 48(1), 207-229.

Kim, J., Salgado, R., and Lee, J. (2002). "Stability analysis of complex soil slopes using limit analysis." J. Geotech. Geoenviron. Eng., 128(7), 546-557.

Kim, J., Salgado, R., and Yu, H. S. (1999). "Limit analysis of soil slopes subjected to pore-water pressures." J. Geotech. Geoenviron. Eng., 125(1), 49-58.

Krabbenhoft, K., Lyamin, A. V., Hjiaj, M., and Sloan, S. W. (2005). “A new discontinuous upper bound limit analysis formulation." Int. J. Numer. Methods Eng., 63(7), 1069-1088.

Li, A. J., Lyamin, A. V., and Merifield, R. S. (2009). "Seismic rock slope stability charts based on limit analysis methods." Comput. Geotech., 36(1-2), 135-148.

Li, A. J., Merifield, R. S., and Lyamin, A. V. (2008). "Stability charts for rock slopes based on the Hoek-Brown failure criterion." Int. J. Rock Mech. Min. Sci., 45(5), 689-700.

Li, A. J., Merifield, R. S., and Lyamin, A. V. (2011). "Effect of rock mass disturbance on the stability of rock slopes using the Hoek-Brown failure criterion." Comput. Geotech., 38(4), 546-558.

Liu, C. H., Jaksa, M. B., and Meyers, A. G. (2008). "Improved analytical solution for toppling stability analysis of rock slopes." Int. J. Rock Mech. Min. Sci., 45(8), 1361-1372.

Liu, C. H., Jaksa, M. B., and Meyers, A. G. (2009). "A transfer coefficient method for rock slope toppling." Can. Geotech. J., 46(1), 1-9.

Lyamin, A. V., and Sloan, S. W. (2002a). "Lower bound limit analysis using nonlinear programming." Int. J. Numer. Methods Eng., 55(5), 573-611.

Lyamin, A. V., and Sloan, S. W. (2002b). "Upper bound limit analysis using linear finite elements and nonlinear programming." Int. J. Numer. Anal. Methods Geomech., 26(2), 181-216.

MOSEK 6.0 [Computer software]. Copenhagen, Denmark, MOSEK ApS.

Müller, L. (1968). "New considerations of the Vajont slide." Felsmechanik Engenieurgeologie., 6(1), 1-91.

Orduna, A., and Lourenco, P. B. (2003). "Cap model for limit analysis and strengthening of masonry structures." J. Struct. Eng., 129(10), 13671375.

Qian, L. X., and Zhang, X. (1995). "Rigid finite element method and its applications in engineering." Acta Mech. Sin., 11(1), 44-50.

Sarma, S. K. (1973). "Stability analysis of embankments and slopes." Geotechnique, 23(3), 423-433.

Sloan, S. W. (1988). "Lower bound limit analysis using finite elements and linear programming." Int. J. Numer. Anal. Methods Geomech., 12(1), $61-77$.

Sloan, S. W. (1989). "Upper bound limit analysis using finite elements and linear programming." Int. J. Numer. Anal. Methods Geomech., 13(3), $263-282$. 
Sloan, S. W., and Kleeman, P. W. (1995). "Upper bound limit analysis using discontinuous velocity fields." Comput. Methods Appl. Mech. Eng., 127(1-4), 293-314.

Yang, F., and Yang, J. S. (2010). "Stability of shallow tunnel using rigid blocks and finite element upper bound solutions." Int. J. Geomech., 10(6), 242-247.

Yang, X. L., Li, L., and Yin, J. H. (2004a). "Seismic and static stability analysis for rock slopes by a kinematical approach." Geotechnique, 54(8), 543-549.

Yang, X. L., Li, L., and Yin, J. H. (2004b). "Stability analysis of rock slopes with a modified Hoek-Brown failure criterion." Int. J. Numer. Anal. Methods Geomech., 28(2), 181-190.
Yang, X. L., and Zou, J. F. (2006). "Stability factors for rock slopes subjected to porewater pressure based on Hoek-Brown failure criterion." Int. J. Rock Mech. Min. Sci., 43(7), 1146-1152.

Yu, H. S., Salgado, R., Sloan, S. W., and Kim, J. M. (1998). "Limit analysis versus limit equilibrium for slope stability." J. Geotech. Geoenviron. Eng., 124(1), 1-11.

Zanbak, C. (1983). "Design charts for rock slopes susceptible to toppling." J. Geotech. Engrg., 109(8), 1039-1062.

Zhang, X. (1999). "Slope stability analysis based on the rigid finite element method." Geotechnique, 49(5), 585-593.

Zhang, X., and Qian, L.X. (1993). "Rigid finite element and limit analysis." Acta Mechanica Sinica, 9(2), 156-162. 